\title{
Muertes violentas y consumo de alcohol
}

\author{
Matos Abella, R.*; Betancourt Pulsan, A.**; Alvarez Cambas, E***; Aces Dunand, S.*; Toirac Perera, S.*. \\ * Médico Legal. ** Médico, Master en Drogodependencias. *** Especialista en Informática.
}

\section{RESUMEN}

Se realizó un estudio descriptivo, retrospectivo y longitudinal para conocer las características de los fallecidos por muertes violentas con cifras positivas de alcohol en sangre registrados por el Departamento Provincial de Medicina Legal de la provincia Guantánamo, en el periodo comprendido entre Enero de 1995 a Diciembre de 1998.

Se utilizaron el libro de defunciones, el registro de archivos de protocolos de necropsias y el libro de registro de alcoholemia del Laboratorio Provincial de Toxicología para caracterizar la muestra escogida ( $n=226$ casos). Se estudiaron las variables: porciento de alcoholemia, edad, sexo, ocupación y lugar de procedencia de los fallecidos, días, meses del año y áreas de ocurrencia, la etiología medicolegal, y las causas básicas y directas de las muertes.

Se obtuvieron como resultados más relevantes que predominó la población joven entre las víctimas, los fines de semana y los meses de verano fueron los más frecuentes en la ocurrencia de los hechos violentos, y la etiología accidental, principalmente los de transito, la colisión y el shock traumático como las variables medico-legales más sobresalientes.

Palabras claves: muerte violenta, alcohol, alcoholemia, medicolegal

\section{SUMMARY}

A descriptive, retrospective and longitudinal study was performed in order to know the characteristics of violent deaths with positive figures of alcohol in blood recorded by the Guantanamo Provincial Department of Legal Medicine between January 1995 to December 1998.

The record of deaths the file of protocols and necropsies as well as the book of records of alcoholemia from the Provincial Laboratory of Toxicology were used to characterised the selected sample ( $n=226$ cases). The variable alcoholemia, age, sex, occupation, and places from where the dead proceed, exact dates (day- month- year), place of deaths, the legal aetiology and the basic and direct causes of deaths were used.

The most relevant results obtained showed that the young population was prominent among the victims. Weekends and summer months were the most frequent in the occurrence of violence principally motorcars, collision and traumatic shock as the must outstanding legal variables.

Key words: violent death, alcohol, alcoholemia, forensic, Cuba.

\section{INTRODUCCIÓN}

E consumo de alcohol data desde los primeros años de la civilización humana, las primeras civilizaciones las utilizaban en sus festividades pues se les atribuían propiedades euforizantes. Sin embargo la historia las asocia también al desorden y los excesos, principalmente a la violencia.

Según se expresa en la literatura mundial, este fenómeno se ha acentuado y acrecentado en este siglo, llegando a considerase como un problema sanitario de gran connotación en el ámbito internacional, y al que se le asocian causas de muertes que pueden ser de origen accidental, suicida u homicida (Came- son, 1975; Carbonell, Piñeiro, Lastre, 1998; Compilación, 1996; Ellis, 1992; Feewerluin, 1982; Gasull et al, 1976; Gonzalez, 1985).

Según estadísticas internacionales en el mundo ocurren en una hora $\mathbf{3 5}$ muertes relacionadas con el alcohol y los accidentes del tránsito, la tercera parte de las muertes violentas por suicidios y homicidios (Montaro, 1997), más de un tercio de los arrestos policiacos y la reducción en 15 años de la expectativa de vida de la población mundial (Boletín, 1995; Montaro, 1997); por lo que se le considera como un factor criminógeno de primerísimo orden y su uso se encuentra íntimamente relacionado con actos delictivos. En forma aproximada el consumo 
de alcohol anual por habitantes mayores de 15 años, asciende aproximadamente a 15 litros en países de Europa Occidental y Oriental, a 10 en Países Escandinavos y Australia, presentándose una cifra exorbitante en EUA, Canadá y América Latina, sobre todo si se tiene en cuenta que estos datos solo reflejan el consumo de bebidas industriales y no toman en cuenta las de fabricación casera (Boletín, 1995; Feewerluin, 1982; Gasull et al, 1976; Gonzalez,1985).

Nuestro país tampoco escapa de los nefastos efectos del uso y abuso del consumo del alcohol, con sus consiguientes repercusiones de carácter altamente nocivo sobre el consumidor y la sociedad. Este problema se ha acentuado en los últimos 25 años y constituye una preocupación para las autoridades en general en nuestro país, particularmente el MINSAP, teniendo en cuenta las influencias alcohólicas como factor desencadenante de muertes violentas, accidentes, homicidios o suicidios, aspecto que está en relación directa con el aumento de las ofertas de bebidas alcohólicas. El análisis de las estadísticas que tratan las influencias alcohólicas en la población de Ciudad de La Habana en 1995 reveló que el $\mathbf{3 2 , 7 \%}$ de los choferes accidentados y el $\mathbf{4 7 , 7 \%}$ de las víctimas de muertes violentas en suicidios y homicidios se encontraban bajo los efectos del alcohol en el momento de ocurrencia de los hechos. Antes de este trabajo existía en nuestra provincia la "impresión" científica de que se ha detectado un incremento de la presencia de alcohol en sangre en las víctimas de muertes violentas con independencia de su etiología médico-legal, lo que nos llama poderosamente la atención. Por lo que se puede apreciar este fenómeno se ha convertido en una epidemia que se ha incrementado a finales de este siglo y a la que se le debe prestar especial importancia (González,1985).

\section{OBJETIVOS}

\section{General:}

Caracterizar desde el punto de vista médico-legal a los fallecidos por muertes violentas con cifras positivas de alcohol en sangre, en el período comprendido desde Enero de 1995 a Diciembre de 1998.

\section{Específicos:}

1. Registrar las muertes violentas con alcoholemia positiva en el período citado.

2. Caracterizar el grupo de estudio en cuanto a: variables epidemiológicas como edad, sexo y ocupación, y según la zona de ubicación geográfica (lugar de procedencia).
3. Describir algunas variables temporales como días y meses del año y las áreas de ocurrencia.

4. Determinar las variables causales como etiología médico legal, causa básica y directa de las muertes.

\section{MÉTODO}

Se realizó una investigación en dependencia de sus objetivos de tipo descriptiva; por su ubicación en el tiempo retrospectiva y longitudinal, de los fallecidos por muertes violentas registrados por el departamento de Medicina Legal de la provincia Guantánamo durante el período comprendido desde Enero de 1995 a Diciembre de 1998.

El universo escogido representó el $100 \%$ de los fallecidos por muertes violentas en el intervalo de tiempo seleccionado. Sin embargo por requerimientos técnicos, de este total de muertes, únicamente son útiles aquellas consideradas como muertes violentas inmediatas, a las que solo es posible aplicarle la prueba de determinación de la concentración de alcohol en sangre. Luego del análisis realizado se seleccionó como muestra a estudiar aquellos fallecidos con cifras positivas de alcohol en sangre.

Se revisaron el Libro de defunciones, el Registro de archivo de protocolos de necropsias, de donde se extrajeron los datos necesarios y el Libro de registro de alcoholemia del Laboratorio de Toxicología, perteneciente al Departamento Provincial de Medicina Legal de Guantánamo, registrando las que resultaron positivas en las muertes violentas analizadas.

Se estudiaron cuidadosamente los protocolos de necropsias y el Libro de registro de defunciones, anotando las variables epidemiológicas (edad, sexo, raza y ocupación); geográficas (lugar de procedencia y municipio de ocurrencia); temporales (hora, día y mes de ocurrencia); causales (etiología médico legal, causa directa y básica de muerte), y otras generales como el área de ocurrencia; para hallar la relación existente entre estos aspectos y las muertes violentas con cifras positivas de alcohol en sangre.

\section{RESULTADOSY DISCUSIÓN}

En la provincia Guantánamo durante el período comprendido entre Enero de 1995 y Diciembre de 1998 ocurrieron un total de $\mathbf{7 0 5}$ muertes violentas inmediatas, de ellas 226 tenían cifras positivas de alcohol en sangre para un $\mathbf{3 2 . 6 \%}$ del total de los fallecidos.

Gráfico No. 1. En él aparecen también los valores de alcoholemia positivas encontrados en los fallecidos

Control samántico: Alcoholemia positiva: aquella que es superior al $\mathbf{2 5 \%} \mathbf{m g} \%$ de alcohol en sangre. Muerte violenta: es aquella que ocurre por alteraciones ajenas al fisiologismo humano. Muerte violenta inmediata: es aquella que ocurre antes de las 24 horas de haberse desencadenado el hecho violento. 
dentro de los que se destaca el rango entre $\mathbf{5 0 - 9 9} \mathbf{~ m \%}$ con 101 casos para un $\mathbf{4 4 . 7 \%}$ de la muestra analizada, lo que se justifica por ser en esta concentración donde predominan los síntomas de agresividad aspecto que incide negativamente en la aparición del hecho violento. Estos resultados coinciden con investigaciones recientes realizadas por Montaro (Montaro, 1997; Mondón, 1997) sobre alcohol y seguridad vial en España.

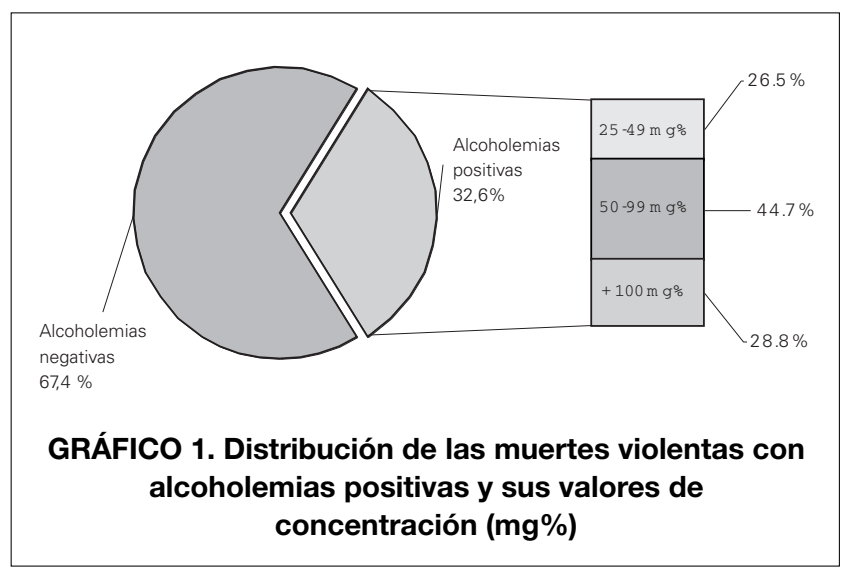

Dentro de las variables sociodemográficas de las víctimas analizadas se constato que predominaba la población joven entre 24-54 años y dentro de estos el rango de $\mathbf{4 5 - 5 4}$ con $\mathbf{4 9}$ casos que representan el $\mathbf{2 1 . 7 \%}$ (Gráfico 2), lo que se fundamenta porque en estas edades es donde existe una mayor independencia económica al estar generalmente incorporados a la actividad laboral, y formar parte de nuevas organizaciones políticas y de masas que permiten una vida social mucho más activa. Estos resultados están en correspondencia con estudios realizados sobre este tema por Carbonell y otros (Carbonell, Piñeiro, Lastre, 1998; Modón, 1997), en la provincia de Las Tunas.

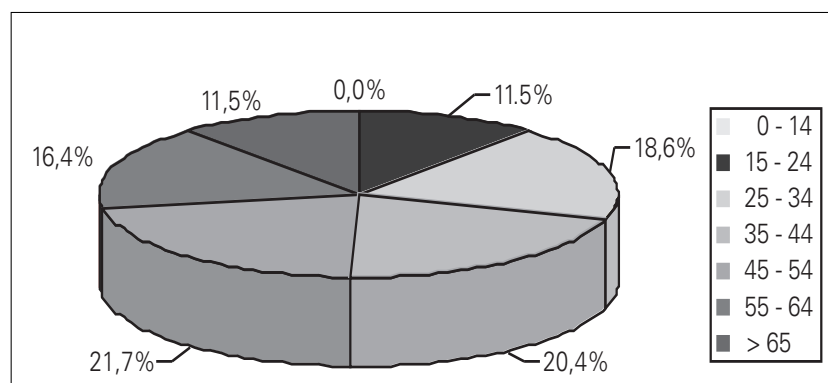

GRÁFICO 2. Distribución de las muertes violentas con alcoholemias positivas según la edad.

En lo relacionado al sexo (Tabla I) sobresalió el masculino con 195 casos para un $\mathbf{8 6 . 2 \%}$ (Carbonell, Piñeiro,Lastre, 1998; Montaro 1997; Mondón, 1997), lo que se justifica por lo arraigado de nuestros prejuicios, costumbres y tradiciones que critican fuertemente el hecho de que las mujeres ingieran o acudan por sí solas a lugares de expendio de bebidas alcohólicas.

\begin{tabular}{|c|c|c|c|}
\hline \multicolumn{3}{|c|}{$\begin{array}{c}\text { Tabla I. Distribución de las muertes violentas y } \\
\text { alcoholemias positivas según sexo. }\end{array}$} \\
\hline \multicolumn{3}{|c|}{ Masculino } & \multicolumn{2}{c|}{ Femenino } \\
\hline No. & $\%$ & No. & $\%$ \\
\hline 195 & 86.2 & 31 & 13.8 \\
\hline
\end{tabular}

La última variable sociodemográfica estudiada fue la ocupación de los fallecidos (grafico 3) dentro de las cuales se destacó la de obrero con $\mathbf{6 9}$ casos con el $\mathbf{3 0 . 5 \%}$, entendiéndose dentro de esta categoría, además de las actividades usualmente conocidas, las agrícolas, forestales, pecuarias y técnico medias. Este fenómeno lo explica la característica de que en nuestro país y en especial en nuestra provincia sea éste el grupo laboral más numeroso, lo que se afianza si tomamos en cuenta el escaso desarrollo fabril e industrial del que dispone nuestro territorio.

El otro grupo numeroso fue el de los desvinculados con $\mathbf{5 7}$ casos para un $\mathbf{2 5 . 2} \%$, tendencia comprensible si sabemos de ante mano que Guantánamo cuenta a escala nacional con la tasa más alta de desocupación (12\%) y que este grupo se caracteriza por desarrollar hábitos de vida poco saludables, dentro de los cuales la ingestión de bebidas alcohólicas adquiere un lugar preponderante.

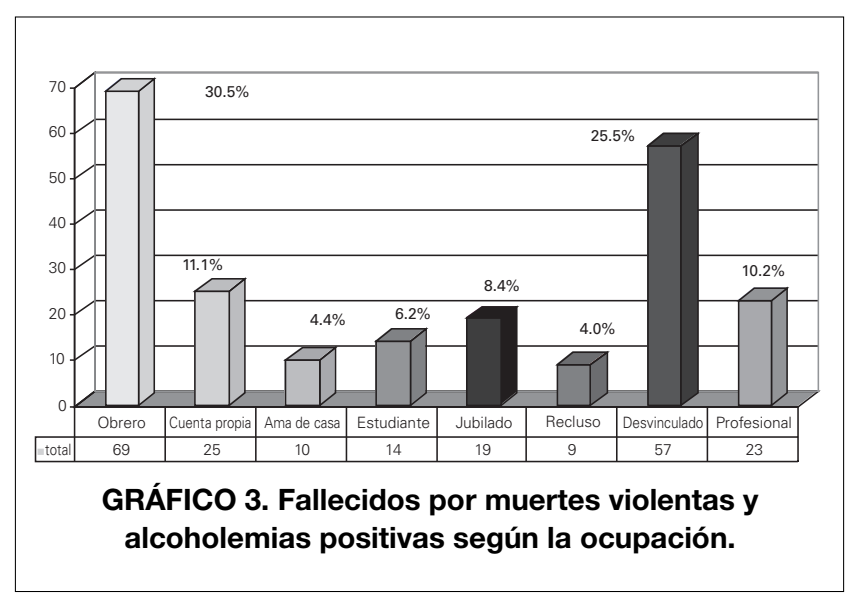

Otra de las variables analizadas fue la de ubicación geográfica de los fallecidos entre la que se encontraba el lugar de procedencia, se comportó según como aparece en la tabla III

Tabla III. Fallecidos por muertes violentas y alcoholemia positiva según lugar de procedencia.

\begin{tabular}{|c|c|c|c|}
\hline \multicolumn{2}{|c|}{ Urbano } & \multicolumn{2}{c|}{ Rural } \\
\hline No. & $\%$ & No. & $\%$ \\
\hline 123 & 54.3 & 103 & 45.7 \\
\hline
\end{tabular}


Como se observa claramente la zona urbana ocupó el lugar predominante con más del $50 \%$ de los casos, lo que se corresponde con la tendencia de mantenerse concentrada una gran cantidad de población en esta área, por encontrarse en la misma un mayor desarrollo de la infraestructura socioeconómica, política y cultural de la provincia, que trae aparejado un incremento de las opciones y centros culturales y recreativos, un desarrollo más acelerado de la circulación de vehículos, y el aumento del expendio de bebidas alcohólicas, factores potencialmente desencadenantes de hechos que conduzcan a la aparición de muertes violentas.

Las muertes violentas estudiadas según las variables temporales relacionadas con la ocurrencia del hecho fatal, según el mes y día en que ocurrieron.

En la tabla IV, se observa que los meses más frecuentes fueron los de julio y agosto con $\mathbf{2 4}$ y $\mathbf{2 5}$ casos $(\mathbf{1 0 . 6} \%$ y $\mathbf{1 0 . 9} \%)$ respectivamente, lo que está avalado por su coincidencia con la etapa vacacional y veraniega, la celebración de fiestas populares y días feriados, los que generalmente vienen acompañados de la venta de bebidas alcohólicas y a los que se le agregan además el aumento de la conglomeración de personas en lugares públicos y recreativos, el aumento del tránsito tanto de vehículos como de peatones, todas ellas condiciones favorables para que ocurra este tipo de muertes.

\begin{tabular}{|c|c|c|c|c|c|c|c|c|c|c|c|}
\hline \multicolumn{8}{|c|}{ Tabla IV. Fallecidos por muertes violentas y alcoholemia } \\
positiva según meses del año.
\end{tabular}

Coincidieron el sábado y domingo como los días más predominantes de la ocurrencia de los fallecimientos, con 41 y 38 casos (18.1\% y 16.9\%), según tabla $V$, lo cual está directamente relacionado a que durante los fines de semana, y como consecuencia del cese de las funciones laborales, se produzca un incremento de las actividades socioculturales y recreativas, la aglomeración de personas en lugares públicos, con el consiguiente expendio de bebidas alcohólicas, factores que al sumarse conducen a que se produzcan violaciones de las normas de comportamiento social lo que condiciona el desencadenamiento de acciones y/o hechos violentos (Montaro 1997; Mondón, 1997).

Tabla V. Fallecidos por muertes violentas y alcoholemia positiva según día de la semana.

\begin{tabular}{|c|c|c|c|c|c|c|c|c|c|c|c|c|c|}
\hline \multicolumn{2}{|c|}{ Lunes } & \multicolumn{2}{|c|}{ Martes } & \multicolumn{2}{l|}{ Miércoles } & \multicolumn{2}{l|}{ Jueves } & \multicolumn{2}{l|}{ Viernes } & \multicolumn{3}{c|}{ Sábado } & \multicolumn{2}{c|}{ Domingo } \\
\hline No. & $\%$ & No. & $\%$ & No. & $\%$ & No. & $\%$ & No. & $\%$ & No. & $\%$ & No. & $\%$ \\
\hline 32 & 14.1 & 29 & 12.9 & 28 & 12.5 & 25 & 10.8 & 33 & 14.7 & 41 & 18.1 & 38 & 16.9 \\
\hline
\end{tabular}

El área de ocurrencia de las muertes más frecuente en este estudio fue la Vía Pública con 101 casos para un $\mathbf{4 4 . 7 \%}$, (gráfico 4) cuestión esta que se respalda por ser en ella donde se producen los accidentes del tránsito, así como muchas reyertas callejeras que terminan en homicidios. Le sigue el área domiciliar con $\mathbf{5 4}$ casos para un $\mathbf{2 3 . 9} \%$, siendo este el lugar propicio o ideal de ocurrencia de los suicidios (Estudios, 15).

Por último los Centros recreativos con $\mathbf{2 9}$ casos $(\mathbf{1 2 . 8} \%)$, donde predominan los homicidios, y la categoría de Otros (dentro de la que se incluyen los centros laborales, áreas agrícolas, extensiones de tierras ociosas, mares, ríos y otros lugares que no están comprendidos en las otras tres categorías) con 42 casos $(\mathbf{1 8 . 6} \%)$ resultaron ser las otras dos variables analizadas

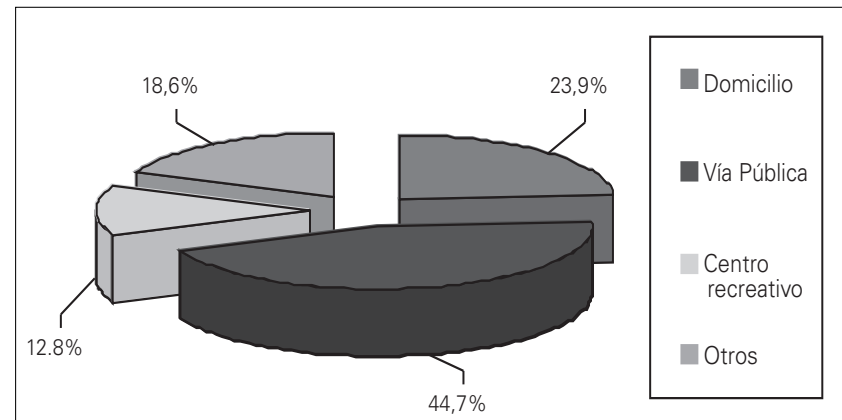

GRÁFICO 4. Distribución de las muertes violentas con alcoholemia positiva según área de ocurrencia.

El otro grupo de categorías analizadas estuvo conformado por las variables causales dentro de las que se ubican la etiología médico legal (gráfico 5) y las causas básica y directa de las muertes (gráficos 6 y 7, respectivamente).

En el primer grupo prevaleció la etiología Accidental con 132 casos para un $\mathbf{5 8 . 4 \%}$, dentro de la que adquiere un mayor grado de representatividad la categoría de Accidentes del tránsito con $\mathbf{8 9}$ casos $(\mathbf{3 9}, \mathbf{4} \%)$, cifras que no son de extrañar si se tiene en cuenta el impacto que produce el alcohol sobre las capacidades psicofísicas de los conductores, afectando su capacidad de reacción y/o la capacidad de controlar la velocidad o distancia, a lo que puede añadírsele el fenómeno del deslumbramiento o pérdida momentánea de visión después de haber observado un objeto luminoso o relampagueante, el cual produce un estado de ceguera transitoria que es capaz de ocasionar la ocurrencia del accidente en un período de tiempo muy breve. Este tipo de efectos puede producirse también aunque con menos frecuencia en el caso de los peatones, y sus consecuencias pueden ser tan graves tanto para unos como para 
otros (Bach, Freixa, 1983; Cameson, 1975; Ellis, 1992; Feewerluin, 1982; Montaro, 1997;Mondón, 1997).

Le siguen en orden de frecuencia los Accidentes comunes con 35 casos $(\mathbf{1 5 \%})$, predominando en estos las caídas y precipitaciones provocadas por la pérdida de las habilidades que produce la ingestión de alcohol, y por último los Accidentes laborales con 9 casos (4\%), haciendo mención de que a pesar que se producen durante la ejecución de funciones de trabajo, ellos son la consecuencia de la infracción de las normas de disciplina laboral.

Al grupo de los accidentes le siguió en orden decreciente el Suicidio con $\mathbf{5 5}$ casos que representan el $\mathbf{2 4 . 3} \%$, influenciado por los síntomas depresivos que produce el alcohol que hacen de este tipo de toxicomanía la responsable de la tercera parte de todos los suicidios a escala mundial (Legra, Aces, 1998)., producto a la fuerza o impulso que este provoca sobre todo en aquellos individuos con trastornos psiquiátricos asociados (A.P.A, DSM, 1989).

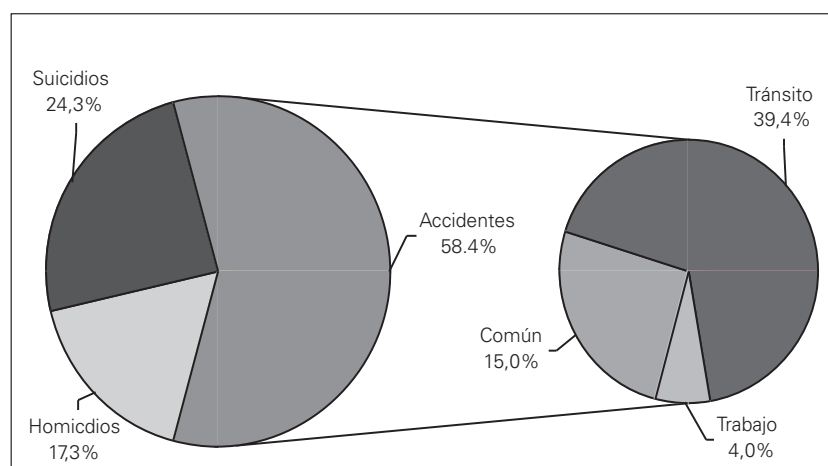

GRÁFICO 5. Fallecidos por las muertes violentas y alcoholemia positiva según etiología médico legal.

Las otras dos variables que restan por discutir son las causas básica y directa de muerte, las que guardan una estrecha relación entre sí pues en dependencia de la forma en que se produzca la muerte así serán los hallazgos anatomopatológicos que se encontrarán en los cadáveres.

Como causas básicas más comunes, la colisión y el atropello ocuparon las primeras posiciones con $\mathbf{4 3}$ y $\mathbf{3 8}$ casos, para un $\mathbf{1 9 \%}$ y $\mathbf{1 6 . 8 \%}$ respectivamente, llevando el peso de la responsabilidad de estas muertes los accidentes del tránsito, considerados la cuarta causa de muerte en el mundo, y como consecuencia de los efectos y alteraciones psicosomáticas que produce la ingestión de bebidas alcohólicas.

En consonancia con esto las causas directas de muertes que predominaron fueron el shock traumático con 59 casos(26.3\%) y el shock hipovolémico con $49 \operatorname{casos}(21.6 \%)$. El primero producido generalmente por las lesiones ocasionadas por la colisión y/o el atropello que por si solas no son capaces de producir la muerte, y al valorarlas en su conjunto nos permiten diagnosticar acertadamente esta entidad; mientras que el segundo tiene como hecho desencadenante generalmente además de los accidentes de tránsito, las heridas producidas por arma blanca y de fuego, las que al lesionar un vaso u órgano importante son capaces de producir la muerte por un sangramiento profuso.

En ambos grupos se incluyeron además de las causas comúnmente conocidas la categoría de Otros, donde agrega un conjunto de causas menores en incidencia pero que fueron también analizadas en nuestra investigación.
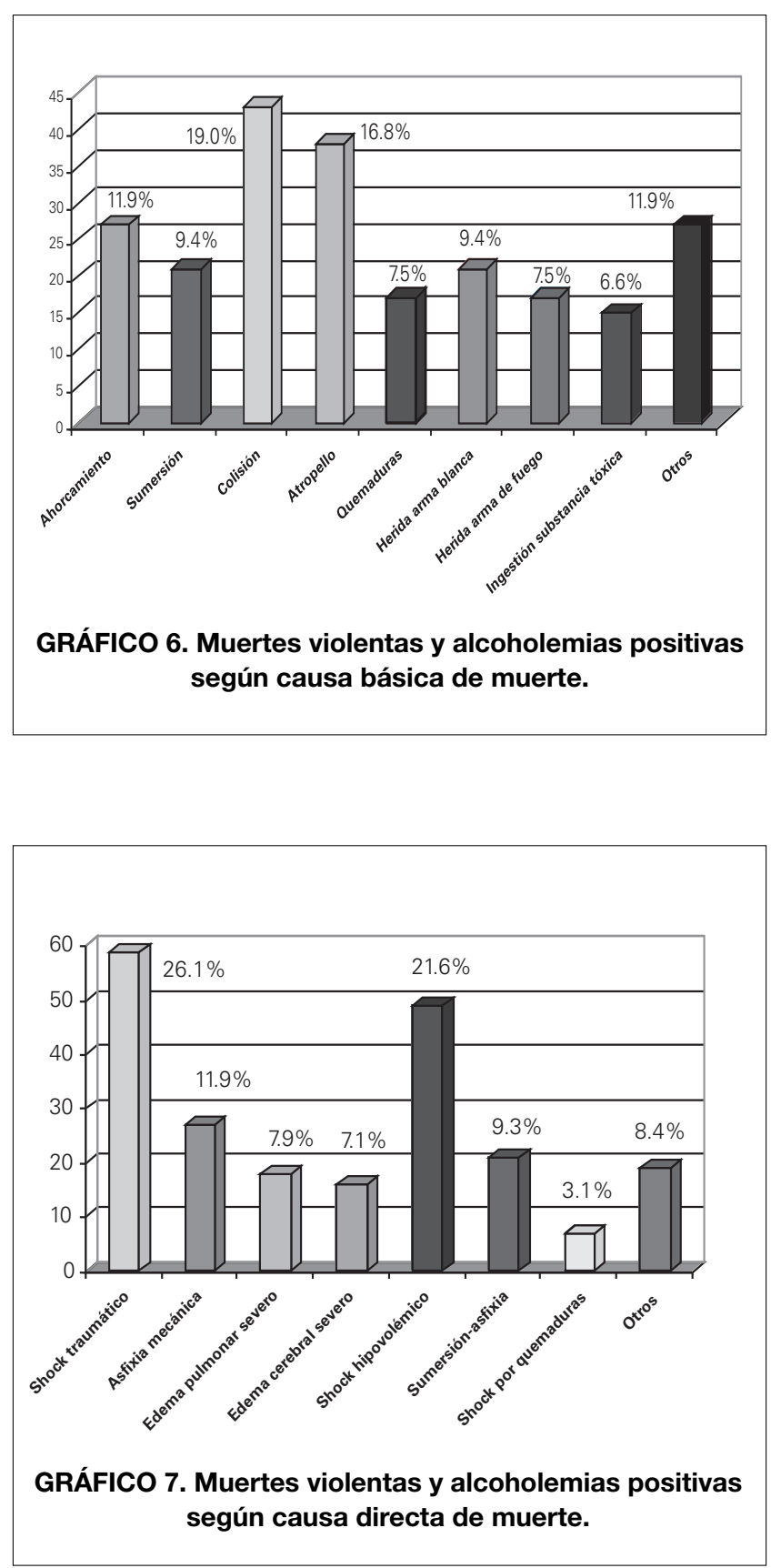


\section{CONCLUSIONES}

En la tercera parte de los fallecidos por muerte violenta se encontró alcoholemia, predominando entre la etiología medico-legal, los accidentes de transito, siendo la colisión y el shock traumático las principales causas básicas y directas, con lo que se coincide con lo que señalan las estadísticas mundiales en cuanto al riesgo de accidentes cuando se consume bebidas alcohólicas, siendo la población joven la de mayor afectación.

\section{BIBLIOGRAFÍA}

1. A. P. A, DSM - III -R. "Manual diagnóstico y estadístico de los trastornos mentale". Barcelona: Ed. Masson, 1989.

2.Bach, L. J., Freixa, F. El alcoholismo. Barcelona: Ed. La Gaya Ciencia, 1983.

3. Boletín Instituto de Medicina Legal Resumen de los trabajos presentados en el Taller de Sociedad, salud y violencia, La Habana, Cuba, 1 de marzo de 1995.

4. Cameson, D. C. "La lucha contra el abuso del alcohol y de las drogas". Crónicas OMS 25 (1): 9-18, Enero 1975 (Sp).

5. Carbonell López, R., Piñeiro González, A. M., Lastre Arrieta, E. "Muertes violentas y alcoholismo". Ponencia Psicohabana'98. La Habana. Cuba, noviembre de 1998.

6. Compilación de artículos de la OPS 1996 La violencia en las Américas: pandemia del siglo XX.
7. Ellis, A. y otros. Terapia racional emotiva con alcohólicos y toxicómanos. Bilbao: Desdu de Brower, 1992.

8. Feewerluin, W. Alcoholismo: abuso, dependencia. Barcelona: Ed. Salvat, 1982.

9. Gasull, Duro, D. A. y otros La enfermedad alcohólica. Barcelona: Químicas Unidas, 1976.

10. González y Méndez, R. La prevención del alcoholismo. Rev. Hosp. Psiq. La Habana 25 (1): 31-39 Ene -Mar. 1985 (Sp).

11. Griffith, E. Tratamientos de alcohólicos, guía para el ayudante profesional. México: Trillos, 1986.

12. Legrá Acosta, M. M., Aces Dunand, S. Comportamiento médico legal del suicidio en la provincia Guantánamo en el período comprendido de 1995-1997. Departamento Provincial de Medicina Legal Guantánamo. Tesis de grado, 1998.

13. Linnoila, M. y otros. "Antecedente familiar de alcoholismo en agresores violentos y agresores impulsivos". Archive of General Psychiatric 46 (7): 613-616,1996.

14. Mondón S, Gual A, Verdaguer J, Colom J "Estudio epidemiológico del consumo de bebidas alcohólicas en accidentes de tráfico los fines de semana". Revista Adicciones 9 (3), 1997

15. Montaro González, L. "Alcohol, drogas, seguridad vial y accidentes de tráfico". Revista Española de Drogodependencia 22 (3), 1997.

16. Rossow, I. "Alcohol-related violence: the impact of drinking patter and drinking context". Addiction 91 (11): 1651- 1661, 1996. 